\title{
Letter
}

\section{Taxonomic status of Panstrongylus herreri Wygodzinsky, 1948 and the number of Chagas disease vectors}

\author{
Jader de Oliveira ${ }^{[1]}$ and Kaio Cesar Chaboli Alevi ${ }^{[2]}$
}

\begin{abstract}
[1]. Laboratório de Parasitologia, Departamento de Ciências Biológicas, Faculdade de Ciências Farmacêuticas, Universidade Estadual Paulista Júlio de Mesquita Filho, Araraquara, SP, Brasil. [2]. Laboratório de Biologia Celular, Departamento de Biologia, Instituto de Biociências, Letras e Ciências Exatas, Universidade Estadual Paulista Júlio de Mesquita Filho, São José do Rio Preto, SP, Brasil.
\end{abstract}

\section{Dear editor:}

A recent Short Communication published by FranzimJunior et al. ${ }^{1}$ in the RSBMT [Franzim-Junior E, Mendes MT, Anhê ACBM, Pelli A, Silva MV, Rodrigues-Junior V, SalesCampos H, Oliveira CJF. The development of Panstrongylus herreri under fluctuating environmental conditions. Rev Soc Bras Med Trop. 2017;50(1):121-5], presented an important study on the one species of the genus Panstrongylus (classified as Panstrongylus herreri Wygodzinsky, 1948). The article included the following statement "P. herreri, also described as $P$. lignarius" and the article by Marcilla et al. ${ }^{2}$ was cited; however, we clarify that the species $P$. herreri was synonymized with P. lignarius (Walker, 1873) by Marcilla et al. ${ }^{2}$. By the Principle of Priority (Article 23 of the International Code of Zoological Nomenclature: http://www.nhm.ac.uk/hosted-sites/ iczn/code/) that states "the valid name of a taxon is the oldest available name applied to it...", Franzim-Junior et al. ${ }^{1}$ presented the development of $P$. lignarius under fluctuating environmental conditions.

In addition, Franzim-Junior et al. ${ }^{1}$ stated "The Triatominae subfamily is comprised of approximately 140 species...", neglecting almost a decade of Triatominae taxonomy, in which nine species were described (Rhodnius zeledoni Jurberg et al., $2009^{3}$, P. martinezorum Ayala, 2009 ${ }^{4}$, Mepraia parapatrica Frias-Lasserre, $2010^{5}, R$. montenegrensis Rosa et al., 20126, R. barretti Abad-Franch et al., 20137 , Triatoma jatai Gonçalves et al., 2013 ${ }^{8}, T$. pintodiasi Jurberg et al., $2013^{9}$ and $R$. marabaensis Souza et al., $2016^{10}, R$. taquarussuensis Rosa et al., 201711), one subspecies and one species were revalidated (T. b. macromelasoma Galvão, $1956^{12}$ and T. bahiensis Sherlock and Serafim, 1967' $)$, and two fossil species were discovered (T. dominicana Poinar, $2005^{14}$ and P. hispaniolae Poinar, $2013^{15}$ ), with a total of 153 species in the Triatominae subfamily distributed in five tribes and 18 genera (Table 1).

Corresponding author: Me. Kaio Cesar Chaboli Alevi.

e-mail: kaiochaboli@hotmail.com

Received 28 March 2017

Accepted 18 May 2017
TABLE 1

Review of the number of species in the subfamily Triatominae.

\begin{tabular}{lcc}
\hline Tribe & Genus & Species (n) \\
\hline Alberproseniini & Alberprosenia & 2 \\
\hline Bolboderini & Belminus & 8 \\
& Bolbodera & 1 \\
& Microtriatoma & 2 \\
& Parabelminus & 2 \\
\hline Cavernicolini & Cavernicola & 2 \\
\hline Rhodniini & Psammolestes & 3 \\
\hline & Rhodnius & 21 \\
\hline Triatomini & Dipetalogaster & 1 \\
\hline & Eratyrus & 2 \\
& Hermanlentia & 1 \\
& Linshcosteus & 6 \\
& Meccus & 6 \\
& Mepraia & 3 \\
& Nesotriatoma & 3 \\
& Panstrongylus & 15 \\
& Paratriatoma & 1 \\
& Triatoma & $\mathbf{7 4}$ \\
\hline \multirow{2}{*}{ Total } & & $\mathbf{1 5 3}$ \\
\hline
\end{tabular}

\section{REFERENCES}

1. Franzim-Junior E, Mendes MT, Anhê ACBM, Pelli A, Silva MV, Rodrigues-Junior V, Sales-Campos H, Oliveira CJF. The development of Panstrongylus herreri under fluctuating environmental conditions. Rev Soc Bras Med Trop. 2017;50(1):121-5

2. Marcilla A, Bargues MD, Abad-Franch F, Panzera F, Carcavallo RU, Noireau F, et al. Nuclear rDNA ITS-2 sequences reveal polyphyly of Panstrongylus species (Hemiptera: Reduviidae: Triatominae), vectors of Trypanosoma cruzi. Infect Genet Evol. 2002;1(3):225-35.

3. Jurberg J, Rocha DS, Galvão C. Rhodnius zeledoni sp. nov. afim de Rhodnius paraensis Sherlock, Guitton \& Miles, 1977 (Hemiptera, Reduviidae, Triatominae). Biota Neotrop. 2009;9(1):123-8.

4. Ayala JM. Una nueva especie de Panstrongylus Berg de Venezuela (Hemiptera: Reduviidae, Triatominae). Entomotropica. 2009;24(3):105-9.

5. Frías-Lasserre D. A new species and karyotype variation in the bordering distribution of Mepraia spinolai (Porter) and Mepraia gajardoi Frías et al (Hemiptera: Reduviidae: Triatominae) in 
Chile and its parapatric model of speciation. Neotrop Entomol. 2010;39(4):572-83.

6. Rosa JA, Rocha CS, Gardim S, Pinto MC, Mendonça VJ, FerreiraFilho JCR, et al. Description of Rhodnius montenegrensis n. $\mathrm{sp}$. (Hemiptera: Reduviidae: Triatominae) from the state of Rondônia, Brazil. Zootaxa. 2012;3478:62-76.

7. Abad-Franch F, Pavan MG, Jaramillo-O N, Palomeque FS, Dale C, Chaverra D, et al. Rhodnius barretti, a new species of Triatominae (Hemiptera: Reduviidae) from western Amazonia. Mem Inst Oswaldo Cruz. 2013;108(Suppl 1):92-9.

8. Gonçalves TCM, Teves-Neves SC, Santos-Mallet JR, Carbajalde-la-Fuente AL, Lopes CM. Triatoma jatai sp. nov. in the state of Tocantins, Brazil (Hemiptera: Reduviidae: Triatominae). Mem Inst Oswaldo Cruz. 2013;108(4):429-37.

9. Jurberg J, Cunha V, Cailleaux S, Raigorodschi R, Lima MS, Rocha DS, et al. Triatoma pintodiasi sp. nov. do subcomplexo $T$. rubrovaria (Hemiptera, Reduviidae, Triatominae). Rev Pan-Amaz Saúde. 2013;4(1):43-56.

10. Souza ES, Von Atzingen NCB, Furtado MB, de Oliveira J, Nascimento JD, Vendrami DP, et al. Description of Rhodnius marabaensis sp. n. (Hemiptera, Reduviidae, Triatominae) from Pará State, Brazil. Zookeys. 2016;621:45-62.
11. Rosa JA, Justino HHG, Nascimento JD, Mendonça VJ, Rocha CS, Carvalho DB, Falcone R, Azeredo-Oliveira MTV, Alevi KCC, de Oliveira J. A new species of Rhodnius from Brazil (Hemiptera, Reduviidae, Triatominae). Zookeys. 2017;675:01-25.

12. Costa J, Correia NC, Neiva VL, Gonçalves TCM, Felix M. Revalidation and redescription of Triatoma brasiliensis macromelasoma Galvão, 1956 and an identification key for the Triatoma brasiliensis complex (Hemiptera: Reduviidae: Triatominae). Mem Inst Oswaldo Cruz. 2013;108(6):785-9.

13. Mendonça VJ, Alevi KCC, Pinotti H, Gurgel-Gonçalves R, Pita S, Guerra AL, et al. Revalidation of Triatoma bahiensis Sherlock \& Serafim, 1967 (Hemiptera: Reduviidae) and phylogeny of the T. brasiliensis species complex. Zootaxa. 2016;4107(2):239-54.

14. Poinar Jr G. Triatoma dominicana sp. n. (Hemiptera: Reduviidae: Triatominae), and Trypanosoma antiquus sp.n. (Stercoraria: Trypanosomatidae), the first fossil evidence of a triatominetrypanosomatid vector association. Vector Borne Zoonotic Dis. 2005;5(1):72-81.

15. Poinar Jr G. Panstrongylus hispaniolae sp. n. (Hemiptera: Reduviidae: Triatominae), a new fossil triatomine in Dominican amber, with evidence of gut flagellates. Palaeodiversity. 2013;6:1-8. 